\title{
Magnetisation switching in a ferromagnetic Heisenberg nanoparticle with uniaxial anisotropy: A Monte Carlo investigation
}

\author{
D. Ledue ${ }^{1}$, P.E. Berche, R. Patte \\ Groupe de Physique des Matériaux, UMR CNRS 6634, Université de Rouen, F-76801 Saint Etienne du Rouvray Cedex, \\ France
}

\begin{abstract}
We investigate the thermal activated magnetisation reversal in a single ferromagnetic nanoparticle with uniaxial anisotropy using Monte Carlo simulations. The aim of this work is to reproduce the reversal magnetisation by uniform rotation at very low temperature in the high energy barrier hypothesis, that is to realize the Néel-Brown model. For this purpose we have considered a simple cubic nanoparticle where each site is occupied by a classical Heisenberg spin. The Hamiltonian is the sum of an exchange interaction term, a single-ion anisotropy term and a Zeeman interaction term. Our numerical data of the thermal variation of the switching field are compared to an approximated expression and previous experimental results on Co nanoparticles.
\end{abstract}

Key words: magnetisation switching, nanoparticle, Monte Carlo simulations PACS: $75.40 . \mathrm{Mg}, 75.60 . \mathrm{Jp}, 75.10 . \mathrm{Hk}$

\section{Introduction}

The exciting field of nanostructured materials offers many challenging perspectives for fundamental research and technological applications. Fundamentally, the investigation of magnetic nanoparticles has already led to a better understanding of magnetic phenomena, such as domain structures [1] and superparamagnetism [2]. From the technological point of view, their potential application in magnetic recording media $[3,4]$ is responsible for a great interest. Indeed, sufficiently small ferromagnetic nanoparticles are single domain $[5,6]$ and are good candidates for high density storage.

\footnotetext{
Email address: denis.ledue@univ-rouen.fr (D. Ledue).

1 Corresponding author.
}

However, too small particles are superparamagnetic even at low temperature and no information can be stored. So an optimal size has to be found for each material.

Moreover high density storage requires a precise knowledge of the magnetisation reversal dynamics. The investigation of the magnetisation switching of individual ferromagnetic nanoparticles driven by an applied field at very low temperature is now possible using the micro-SQUID technique $[7,8,9,10]$. By waiting time, switching field and telegraph noise measurements on an ellipsoidal Co particle, these authors concluded that the magnetisation reversal can be described by thermal activation over a single-energy barrier as proposed by Néel and Brown [11,12]. On the other hand, because of their small size, nanoparticles are good candi- 
dates for numerical investigations in which they can be studied with a realistic size. Magnetisation switching in small ferromagnetic nanoparticles has been previously investigated by Monte Carlo (MC) simulations on Ising $[13,14,15]$ or Heisenberg models $[16,17]$. For highly anisotropic systems, it has been shown that the reversal process is not uniform but rather nucleation-like. Analytical arguments and MC results have been compared with good agreement $[14,15]$.

The simulation of the uniform rotation requires models with continuous degrees of freedom, like the classical Heisenberg model. Then, by varying the anisotropy strength or the particle size, it is possible to investigate the crossover from uniform rotation to nucleation [16]. In this paper, we wish to reproduce the reversal magnetisation by uniform rotation in a single ferromagnetic nanoparticle with uniaxial anisotropy at very low temperature. In our case where the spins are strongly coupled and the energy barrier is large compared to $k_{B} T$, that is very large metastable lifetime, the Langevin dynamics formalism [12] would require a huge computational effort. Indeed, for such systems, this method is restricted to timescales of the oredr of a few nanoseconds [18]. So we have chosen Monte Carlo simulations which do not take into account the precession process but allow to describe considerably larger timescales. We have used the standard Metropolis algorithm. Unlike previous works devoted to the lifetime of the metastable state $[16,17]$, we are interested here in the switching field which depends on the temperature but also on the field sweeping rate. Actually, we aim to check the scaling behaviour predicted by approximated calculations $[19,20]$ in the framework of the Néel-Brown model. Our results will be compared to recent experimental measurements $[7,8]$ which are in good agreement with this scaling behaviour. We also wish to precise the limits of validity of this approximated expression in terms of temperature and field sweeping rate. The study is carried out for several particle sizes and anisotropy constant values. In Section 2, we briefly remind the main predictions of the Néel-Brown theory and also derive results concerning the switching field. The model and the simulation technique are presented in Section 3. The results are discussed in Section 4 and a conclusion is given in Section 5 .

\section{Magnetisation switching of a single nanoparticle}

The stable configurations of a ferromagnetic particle of radius $R$ depend on the two ratios $R / \lambda$ and $R / \delta$ where $\lambda \sim \sqrt{J} / M_{s}$ is the exchange length and $\delta \sim \sqrt{J / D}$ is the domain wall width $\left(J, M_{s}\right.$ and $D$ are, respectively, the exchange interaction, the spontaneous magnetisation and the uniaxial anisotropy constant per atom). More precisely, the nanoparticle is single domain (with all spins aligned) if its radius is smaller than a critical value [5]. Such a nanoparticle exhibits two ground states of opposite magnetisation along the easy axis, for example the $x$ axis. Under an applied field $\mathbf{H}$ in the opposite direction of the particle's magnetisation, the magnetisation is expected to reverse by uniform rotation, that is, the spins remain parallel during the reversal. According to the Stoner-Wohlfarth model [21] and its generalisation $[22]$ the energy barrier which is only due to the anisotropy of the system can be written, at $0 \mathrm{~K}$, as

$\Delta E(H)=\Delta E_{0}\left(1-\frac{H}{H_{s w}^{0}}\right)^{\alpha}$

where $\Delta E_{0}$, the anisotropy energy barrier in zero field of a $N$-spin particle, is given by

$\Delta E_{0}=N D S^{2}$

( $S$ is the spin value). The exponent $\alpha$ depends on the direction of the applied field: $\alpha$ is equal to 2 when the field is along the anisotropy axis or perpendicular to it and $\alpha \approx 3 / 2$ else [7]. $H_{s w}^{0}$, the field for which the energy barrier vanishes, is the switching field at $0 \mathrm{~K}$. Assuming that the intensity of the atomic moment is related to the spin value by $m=g \mu_{B} S$ leads to

$H_{s w}^{0}=\frac{2 D S}{g \mu_{0} \mu_{B}}$.

If $k_{B} T<<\Delta E(H)$, only the up and down magnetized states along the $x$ axis can be observed and if $k_{B} T<<J$, Eqs. (1), (2) and (3) remain roughly 
valid. From the Néel-Brown theory [11,12], the lifetime of the metastable state can be expressed by a thermally activated expression

$\tau(T, H)=\tau_{0} \exp \left(\Delta E(H) / k_{B} T\right)$

where the prefactor $\tau_{0}$ is usually supposed constant for simplicity [7]. Actually, it is the transfer of the thermal energy of the lattice to the system of coupled spins which is responsible for the rotation of the magnetic moments through the equatorial $y-z$ plane. So $\tau_{0}$ depends on the temperature but also on the applied field $[11,12,23,24]$.

The magnetisation reversal can be investigated by increasing the applied field over time and measuring the switching field. Since the probability $P(t)$ that the magnetisation has not switched after a time $t$ decreases as the temperature increases $(P(t)=\exp [-t / \tau(T, H)])$, the switching field decreases as the temperature increases. On the other hand, the switching field increases with the field sweeping rate $v=\frac{d H}{d t}$. Since the magnetisation reversal by thermal activation is a stochastic process, the covered trajectory in the phase space is different from an experiment to another, so the switching field is varying and one has to deal with a switching field distribution. For small enough field sweeping rates, this distribution is given by [19]

$p(T, H)=\frac{1}{\tau(T, H) v} \exp \left[-\int_{0}^{H} \frac{d H^{\prime}}{\tau\left(T, H^{\prime}\right) v^{\prime}}\right]$.

It is the product of the probability $\frac{1}{\tau(T, H) v}$ that the reversal occurs in the interval $[H, H+d H]$ by the probability that the reversal has not yet occured. From the Taylor's development of $p(H)$ around its maximum, one can deduce the mean switching field by

$H_{s w}(T, v) \approx H_{s w}^{0}\left[1-\left(\frac{k_{B} T}{\Delta E_{0}} \ln \left(\frac{c T}{v \varepsilon^{\alpha-1}}\right)\right)^{1 / \alpha}\right]$

where $c=k_{B} H_{s w}^{0} /\left(\alpha \tau_{0} \Delta E_{0}\right)$ and $\varepsilon=1-$ $H_{s w} / H_{s w}^{0}[19,20]$. Then, assuming that the standard deviation is roughly equal to the half-width of the distribution, one obtains $[19,20]$

$\sigma(T, v) \approx \frac{H_{s w}^{0}}{\alpha}\left(\frac{k_{B} T}{\Delta E_{0}}\right)^{1 / \alpha}\left[\ln \left(\frac{c T}{v \varepsilon^{\alpha-1}}\right)\right]^{(1-\alpha) / \alpha}$
This quantity increases with temperature and with the field sweeping rate.

\section{Model and simulation technique}

\subsection{Model}

Our model consists of a simple cubic lattice included in a sphere of radius $R$ with free boundary conditions. Each site is occupied by a classical Heisenberg spin $\mathbf{S}_{i}=\left(S_{i}^{x}, S_{i}^{y}, S_{i}^{z}\right)$ of modulus $S$ in order to reproduce uniform rotation and to investigate the effect of the anisotropy strength. The hamiltonian $H$ is given by

$H=-J \sum_{<i, j>} \mathbf{S}_{i} . \mathbf{S}_{j}-D \sum_{i}\left(S_{i}^{x}\right)^{2}-\mathbf{H} . \sum_{i} \mathbf{S}_{i}$

where $J>0$ is the ferromagnetic exchange interaction limited to the nearest neighbors and $D>0$ is the anisotropy constant on each site. The first term favors alignment of all spins and the second one favors alignment along the $x$ axis (up or down). The last sum represents the interaction with the applied field. Here, we have considered an applied field along the $x$ axis: $\mathbf{H}=-H \hat{\mathbf{e}}_{x}$ where $\hat{\mathbf{e}}_{x}$ is the unit vector and $H>0$. Then, the ground state corresponds to all spins being antiparallel to the unit vector $\hat{\mathbf{e}}_{x}$ whereas the metastable state corresponds to all spins pointing up in the $x$ direction. We have to mention that we have taken here the same exchange interactions, anisotropy constants and anisotropy axis for the core and the surface of the nanoparticle although surface anisotropy is usually considered as radial and stronger. Since our calculations are restricted to very small nanoparticles, much smaller than the critical size to be single-domain in the remanent state [25], we have neglected dipolar interactions. The magnetisation per spin of the particle is defined by

$$
\mathbf{M}=\frac{1}{N} \sum_{i=1}^{N} \mathbf{S}_{i}
$$




\subsection{Simulation technique}

The numerical procedure is the importancesampling MC method $[26,27,28]$. In our simulations, we have used the standard Metropolis algorithm [29]. Each simulation is performed at a given temperature $T$. The particle is initially magnetized in the $x$ direction by applying a strong field $\mathbf{H}_{\mathbf{0}}=H_{0} \hat{\mathbf{e}}_{x}\left(H_{0}>0\right)$ during $n$ MC steps (a MC step (MCS) corresponds to the scan of all spins once, trying a rotation for each). Then, the antiparallel applied field is ramping by small jumps $\delta H: \mathbf{H}_{p}=-p \delta H \hat{\mathbf{e}}_{x}$. Since the field is kept constant during $n$ MCS, the field sweeping rate is given by $v=\delta H / n$. The simulation is stopped when the magnetisation reversal is observed (the arbitrary chosen criterion is $M_{x}<-0.8 S$ ) and the switching field value is stored. For each temperature and each value of the field sweeping rate, a relatively large number of simulations $n_{s}$ has to be performed to get a reliable estimate of the mean switching field $H_{s w}(T, v)$. This has been done using a parallel version of the code. The error for $H_{s w}(T, v)$ will be given by $\sigma(T, v) / \sqrt{n_{s}}+\delta H / n_{s}$ where $\sigma(T, v)$ is the numerical estimate of the standard deviation of the switching field distribution.

Starting from the initial state magnetized in the $x$ direction, a uniform rotation of the magnetisation of an angle $\theta$ produces, using Eq. (8), an energy variation

$$
\delta E=N S(1-\cos \theta)[D S(1+\cos \theta)-H]
$$

where there is no exchange energy. Simulating this mechanism by a single spin rotation (SSR) algorithm implies an increase of the exchange energy at each individual rotation of $\theta$. The corresponding energy variation of the system is

$$
\delta E_{\mathrm{SSR}}=S(1-\cos \theta)[z J S+D S(1+\cos \theta)-H]
$$

where $z$ is the coordination number. Since the acceptance of the SSR requires $\delta E_{\mathrm{SSR}} \leq 0$ at $0 \mathrm{~K}$, no individual rotation can occur at $0 K$ if $H<$ $H_{\mathrm{SSR}}^{c}=z J S+D S(1+\cos \theta)$ and, consequently, no uniform rotation of all spins. This clearly means that the algorithm fails at $0 K$ since it gives a switching field value larger than the field which produces the flip of a surface $\operatorname{spin}(z=1)$, that is $H=J S$ which has nothing to do with the ex- pected value $H_{s w}^{0}$. At very low temperature and small applied field as in our simulations (that is $\delta E_{\mathrm{SSR}}>0$ ), SSR has a very small occurence probability and uniform rotation itself can require a very large number of MCS. So, the switching field at very low temperature will be overestimated except for small enough field sweeping rates. There is a temperature range and field sweeping rate range for which the algorithm dynamics is suitable for the investigation of the uniform rotation.

\section{Numerical results}

The spin value has been fixed to $S=1$ for all nanoparticles. The physical parameters $N, J$ and $D$ have to be chosen carefully in order to compare our results with previous experimental ones with a reasonable computational effort, that is small sizes. Actually, the magnetisation reversal process depends, as the stable states, on the two ratios $R / \lambda$ and $R / \delta$. In their experiments, W. Wernsdorfer et al. [7] have investigated a single domain Co nanoparticle with a radius of $(12.5 \pm 2.5) \mathrm{nm}$ which yields $R / \lambda \simeq 1.5$ and $R / \delta \simeq 0.7$. Here, we have considered a $33-$ spin nanoparticle $(R=2 a, a$ is the lattice parameter) with $J=k_{B}$ and $D=0.1 J$ so $R / \lambda \simeq 1$ and $R / \delta \simeq 0.3$. These values are of the same magnitude as the experimental ones. Actually, in our model, we have underestimated the exchange interaction of a factor 250 in comparison to Co which decreases the two characteristic lengths $\lambda$ and $\delta$ and allow us to investigate very small nanoparticles. The choice of $J$ has only effect on the simulated temperatures since we have imposed the low temperature condition $k_{B} T<<$ $J\left(0.01 \leq k_{B} T / J \leq 0.1\right)$. It has to be mentioned that the quality factor $Q=(\lambda / \delta)^{2}$ which defines the hardness of the ferromagnetic material is equal to 0.09 in our model, of the same magnitude as the Co value 0.2. The mean switching field, at a given temperature and for a given field sweeping rate, was obtained by averaging over 100 different realisations.

Firstly, we checked that our definition of the field sweeping rate $v=\delta H / n$ is reliable; then, we have determined the thermal variation of the switching 


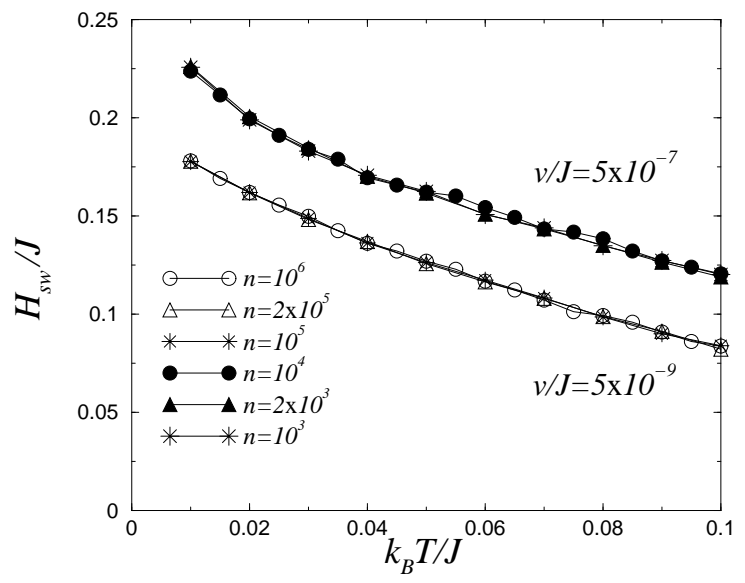

Fig. 1. Thermal variation of the mean switching field for $\delta H / n=5 \times 10^{-7} J$ and $5 \times 10^{-9} J$ for three different values of $(n, \delta H)(N=33$ spins and $D=0.1 J)$. The error bars are smaller than the symbols.

Table 1

Field sweeping rate versus the number of MCS for $\delta H=$ $5 \times 10^{-3} \mathrm{~J}$.

\begin{tabular}{|l|l|l|l|l|l|l|l|l|l|}
\hline$n\left(\times 10^{3}\right)$ & 5 & 10 & 50 & 100 & 500 & 1000 & 5000 & 10000 & 100000 \\
\hline$v / J\left(\times 10^{-6}\right)$ & 1 & 0.5 & 0.1 & 0.05 & 0.01 & 0.005 & 0.001 & 0.0005 & 0.00005 \\
\hline
\end{tabular}

field of a given particle with different values of $\delta H$ and $n$ such that $\delta H / n$ is kept constant. In Fig. 1, we have plotted the two cases $\delta H / n=5 \times 10^{-7} J$ and $5 \times 10^{-9} \mathrm{~J}$. It can be seen in both cases that the three curves corresponding to different numbers of MCS are almost identical indicating that $\delta H / n$ is a reliable definition for the field sweeping rate. The fluctuations of the data are smaller for $v=$ $5 \times 10^{-9} \mathrm{~J}$. In the following, we have fixed $\delta H=$ $5 \times 10^{-3} J$ and only $n$ is varying (Table 1 ).

\subsection{Temperature and field sweeping rate dependence of the reversal field}

We present here our numerical results concerning a nanoparticle of $N=33$ spin with an anisotropy constant $D=0.1 J$. In Fig. 2, we have plotted the time evolution of the modulus and the components of the magnetisation per spin for $k_{B} T / J=0.01$ and 0.1 . For $k_{B} T / J=0.01$, the thermal fluctuations of the modulus are very small and the uniform rotation is observed (the modulus
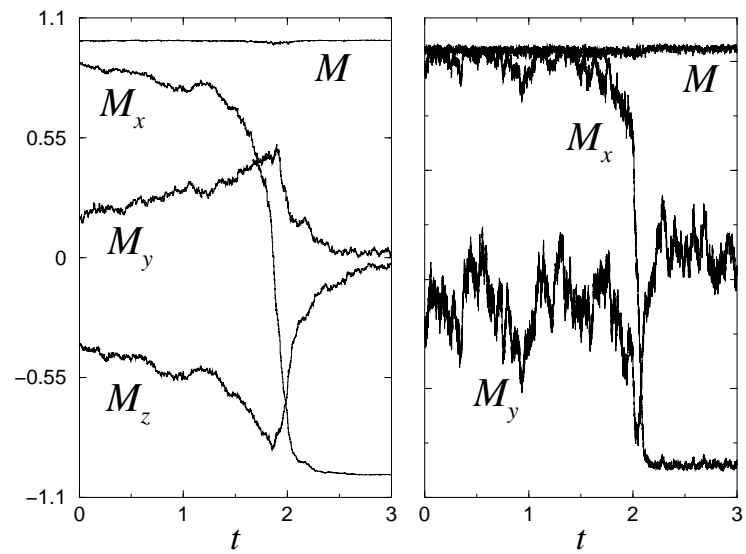

Fig. 2. Time dependence in $10^{4} \mathrm{MCS}$ of the modulus and the components of the magnetisation per spin at $k_{B} T / J=0.01$ (left side) and $k_{B} T / J=0.1$ (right side) $(N=33$ and $D=0.1 J)$. For reason of clarity, the $M_{z}$ component is not shown on the right side. Note that we have plotted the time evolution for only three values of the field.

is roughly constant during the reversal). The reversal duration is about $4 \times 10^{4} \mathrm{MCS}$ which means that the reversal dynamics is slow. For $k_{B} T / J=$ 0.1 , the reversal is still uniform but thermal fluctuations are visible. Many reversal attempts can be seen before the reversal itself whose duration is significantly reduced to about $4 \times 10^{3}$ MCS. We can conclude that these parameters allow to be in the uniform rotation regime unlike the case of larger anisotropy constant, as it will be seen later.

The probability distribution of the switching field for 5000 samples is plotted in Fig. 3 at $k_{B} T / J=0.01$ and 0.1 for two different field sweeping rates $v / J=10^{-6}$ and $10^{-7}$. We clearly see the gaussian shape of the distribution whose width proportional to $\sigma$ increases with $v$ and $T$ in agreement with Eq. (7).

The field sweeping rate dependence of the mean switching field for several temperatures is shown in Fig. 4. An almost logarithmic dependence at small sweeping rate can be observed as in Ref. 7 whose experimental results are shown for comparison. In these experiments the mean switching field has been measured over several hundred cycles.

To check the validity of Eq. (6), one can plot the mean switching field values versus 

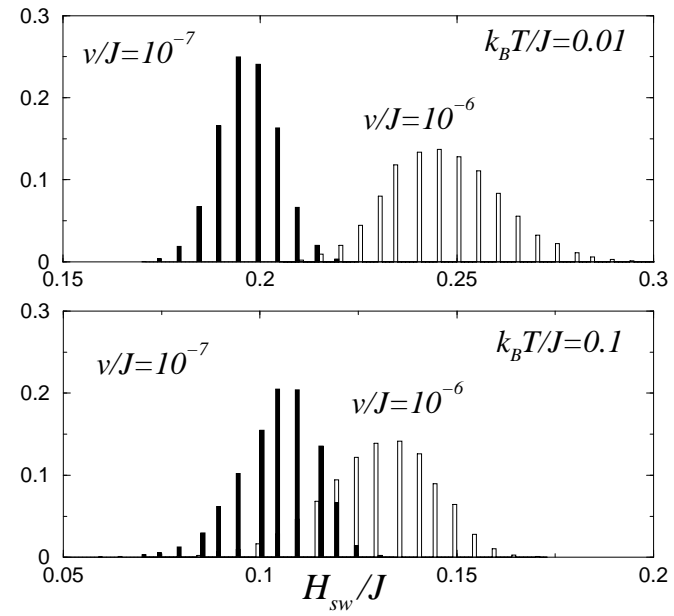

Fig. 3. Probability distribution of the switching field at $k_{B} T / J=0.01$ and 0.1 for $v / J=10^{-6}$ and $10^{-7}(N=33$ and $D=0.1 J)$. Each histogram is obtained from 5000 samples.

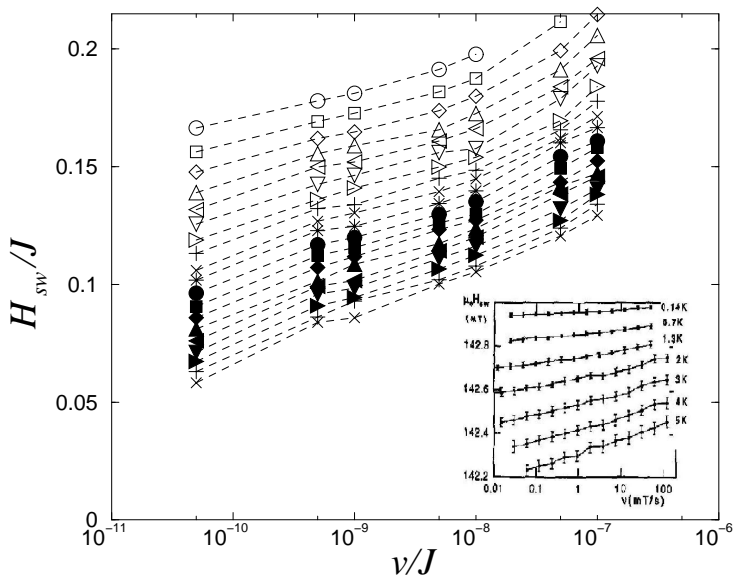

Fig. 4. Field sweeping rate dependence of the mean switching field of a 33-spin particle with $D=0.1 J$ for different temperatures $0.01 \leq k_{B} T / J \leq 0.1$ (increasing temperature from the top to the bottom). Insert: Experimental results of Wernsdorfer et al [7].

$\left[k_{B} T \ln \left(\frac{c T}{v \varepsilon}\right)\right]^{1 / 2}$ where $\varepsilon$ depends on $H_{s w}$. If the scaling behaviour is satisfied, all data points should collapse on a single straight line by choosing correctly the constants $H_{s w}^{0}$ and $c$. Then, a self-consistent fit is obtained when the vertical axis intercept is the chosen value $H_{s w}^{0}$. From Eq. (6) , the slope is $-H_{s w}^{0} /\left(\Delta E_{0}\right)^{1 / 2}$ from which it is possible to extract an estimate of $\Delta E_{0}$. Putting back $H_{s w}^{0}$ and $\Delta E_{0}$ in the expression of $c$ provides

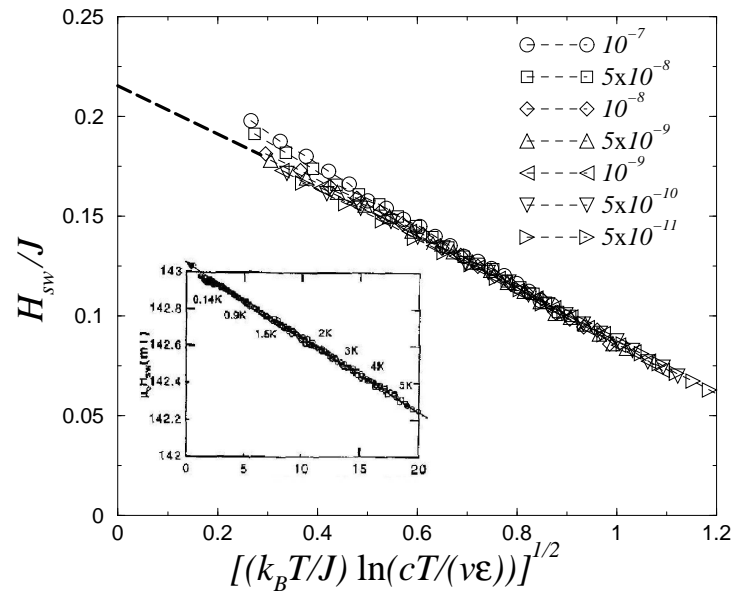

Fig. 5. Scaling plot of the mean switching field values of a 33 -spin particle with $D=0.1 J$ for $5 \times 10^{-11} \leq v / J \leq 10^{-7}$ and $0.01 \leq k_{B} T / J \leq 0.1$. The dashed line shows the mean linear fit. Insert: experimental results of Wernsdorfer et al [7].

a numerical value for $\tau_{0}$. Such a fit is shown in Fig. 5.

Only the curves corresponding to the smallest field sweeping rates $\left(v / J \leq 10^{-8}\right)$ collapse on a single straight line. Numerical data associated to higher sweeping rates move away from the scaling behaviour as the temperature decreases. This deviation is attributed to the inefficiency of the SSR procedure at very low temperature (see Section 3.2) because our numerical switching field values can be larger than $H_{s w}^{0}$ which is not physical. No deviation due to thermal fluctuations has been noticed even when $k_{B} T / J \rightarrow 0.1$. The results of the best fit obtained with $c / k_{B}=10^{-3}$ and $H_{s w}^{0}=$ $0.215 J$ are reported in TABLE 2 . The vertical axis intercept is consistent with the value used to perform the fit but is slightly higher than the expected one $H_{s w}^{0} \simeq 0.2 J$ (Eq. (3) with $g \mu_{0} \mu_{B}=1$ ). Our estimate of the energy barrier in zero field $2.8 \mathrm{~J}$ is lower than the expected value $\Delta E_{0} \simeq 3.3 \mathrm{~J}$ (Eq. (2)).

\subsection{Influence of the particle size on the reversal field}

Since the magnetisation reversal mechanism is very sensitive to the system size, we have studied 
Table 2

Parameters of the fits and estimates of the switching field at $0 \mathrm{~K}$ and the energy barrier in zero field for two particle sizes and $D=0.1 J$. The axis intercept corresponds to $H_{s w}^{0} / J$ and the slope to $-\left(H_{s w}^{0} / J\right) /\left(\Delta E_{0} / J\right)^{1 / 2}$.

\begin{tabular}{|l|l|l|}
\hline$N$ & 33 & 123 \\
\hline$H_{s w}^{0} / J$ & 0.215 & 0.215 \\
$c / k_{B}$ & $10^{-3}$ & $10^{-4}$ \\
axis intercept & 0.219 & 0.215 \\
slope & -0.132 & -0.080 \\
$\Delta E_{0} / J$ & 2.8 & 7.2 \\
$\tau_{0}$ & 40 & 150 \\
\hline
\end{tabular}

the influence of the number of spins on the mean switching field variation in the case $D=0.1 \mathrm{~J}$.

For $N=7(R=a)$, the time dependence of the modulus and the components of the magnetisation exhibits no significant difference from the case $N=33$ except an increase of the thermal fluctuations. Indeed, for a given temperature, thermal fluctuations increase as the size of the system decreases. A consequence is that the reversal duration is reduced to about $6 \times 10^{3} \mathrm{MCS}$ and $10^{3} \mathrm{MCS}$ at $k_{B} T / J=0.01$ and 0.1 respectively. For $N=123$ $(R=3 a)$, the two ratios $R / \lambda \simeq 1.5$ and $R / \delta \simeq 0.4$ are closer to the experimental values; in that case, we have observed again that the reversal is uniform which agrees with experimental results [7].

The plot of the mean switching field values versus $\left[k_{B} T \ln \left(\frac{c T}{v \varepsilon}\right)\right]^{1 / 2}$ is drawn in Fig. 6 for $N=7$, 33 and 123.

For $N=123$, using the same procedure as in the previous section, we observed similar results as for $N=33$. A self-consistent fit can be obtained with the same value of $H_{s w}^{0}$ as for $N=33$ in agreement with the fact that $H_{s w}^{0}$ should not depend on the particle size (TABLE 2). The extracted energy barrier in zero field is significantly lower than the expected value $\Delta E_{0} \simeq 12.3 \mathrm{~J}$ again.

In the case $N=7$, the curvature observed as the temperature increases corresponds to the transition towards the superparamagnetic regime. Indeed, the particle is still strongly magnetized at these temperatures (the time average of the modulus of $\mathbf{M}$ at $k_{B} T / J=0.1$ is $0.934 S$ ), so the zero mean switching field (actually $\delta H$ ) obtained for

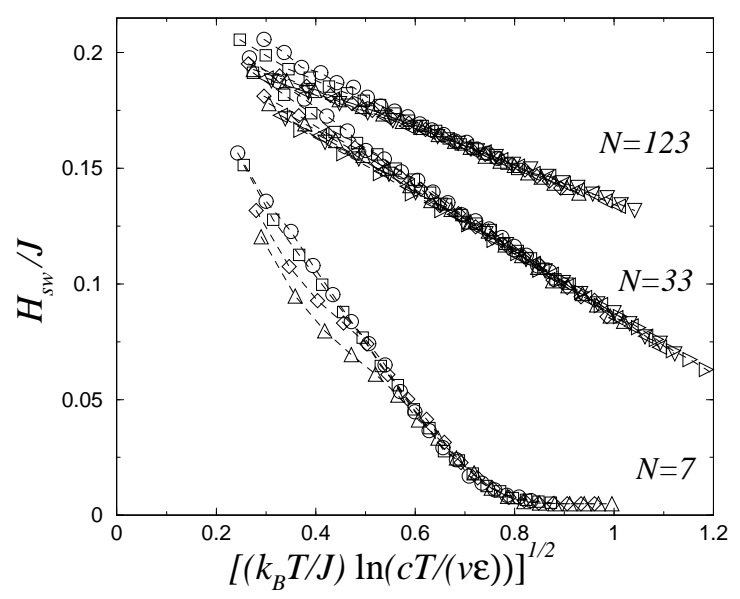

Fig. 6. Scaling plot of the mean switching field values of a 7 -spin, a 33 -spin and a 123-spin particle with $D=0.1 J$ and $0.01 \leq k_{B} T / J \leq 0.1$. The values of the field sweeping rate are $5 \times 10^{-9} \leq v / J \leq 10^{-7}$ for $N=7,5 \times 10^{-11} \leq v / J \leq 10^{-7}$ for $N=33$ and $5 \times 10^{-10} \leq v / J \leq 10^{-7}$ for $N=123$.

the smallest field sweeping rates means that the magnetisation spontaneously reverses in zero field. At low temperature, the data do not lie on a single straight line. The deviation observed for $v / J=$ $5 \times 10^{-9}$ and $10^{-8}$ is attributed to unlikely single flips of spins near the particle boundary (the coordinence number is only 1 for 6 spins) which induce the magnetisation reversal earlier as expected. For this reason, we did not perform simulations with smaller field sweeping rates. This deviation has not been observed for $N=33$ and 123 because surface effects are much less pronounced.

\subsection{Influence of the anisotropy constant on the reversal field}

The ratio $D / J$ is also supposed to play an important role in the magnetisation reversal process. Here, we have investigated for a 33-spin nanoparticle the effect of increasing the anisotropy strength $(D=0.2 J, 0.3 J$ and $J$ ) to evidence the transition to a non uniform rotation regime and to study its consequence on the switching field variation.

For $D=0.3 J(R / \delta \simeq 0.5)$, the reversal is not perfectly uniform since the jump of the modulus of the magnetisation during the reversal is about 

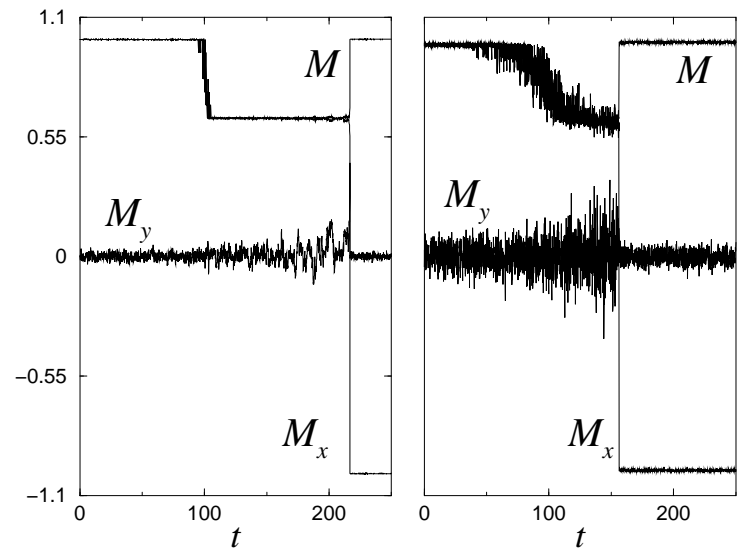

Fig. 7. Time dependence in $10^{4}$ MCS of the modulus and the $M_{x}, M_{y}$ components of the magnetisation per spin at $k_{B} T / J=0.01$ (left side) and $k_{B} T / J=0.1$ (right side) $(N=33$ and $D=J)$.

$10 \%$ at $k_{B} T / J=0.01$ (for $D=0.2 J$, the jump is around $6 \%$ ). The reversal duration for $D=0.3 \mathrm{~J}$ at $k_{B} T / J=0.01$ is about $2 \times 10^{4} \mathrm{MCS}$ which is of the same order of magnitude as for $D=0.1 J$ and $0.2 \mathrm{~J}$.

For $D=J(R / \delta \simeq 0.9)$ the material of our model is harder than Co (the quality factor is about 0.8). As it can be seen in Fig. 7, the reversal is clearly not uniform (the jump is about $36 \%$ ). Because of strong anisotropy, the spins behave roughly as Ising spins and the magnetisation is always along the $x$ axis except short time scale fluctuations. This was not the case for $D=0.1 J$ (see Fig. 2). Actually the spins near the surface of the nanoparticle firstly flip whereas the other spins with higher coordination number flip later, that is for a larger value of the field. At $k_{B} T / J=0.01$, the magnetisation reversal occurs in only two steps indicating that the nanoparticle behaves as a core surrounded by a surface shell. At $k_{B} T / J=0.1$, the reversal is more continuous due to thermal activation.

To check the validity of Eq. (6) for $D=0.2 \mathrm{~J}$ and $0.3 \mathrm{~J}$, we have plotted the mean switching field values using the same procedure as in the previous sections. Although the reversal is not perfectly uniform, it is possible to put the data points corresponding to $v / J \leq 10^{-8}$ on a single straight line (Fig. 8). As for $D=0.1 \mathrm{~J}$, the scaling behaviour is satisfied for all temperatures. The results of the

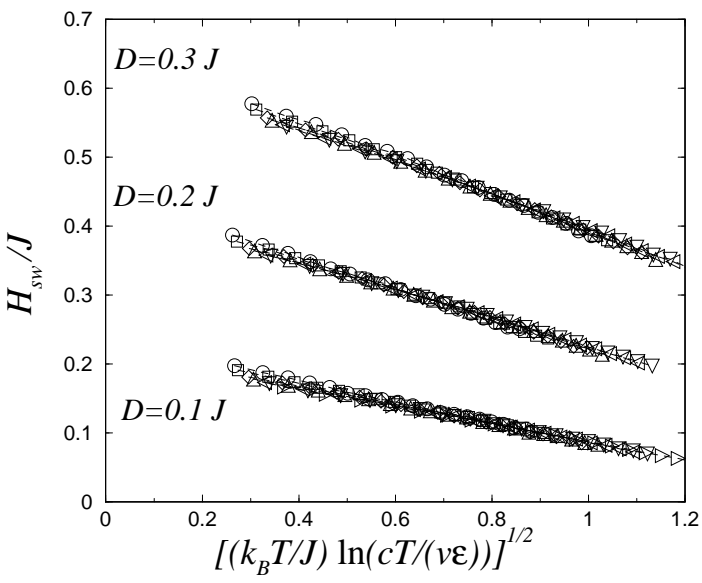

Fig. 8. Scaling plot of the mean switching field values of a 33-spin particle with $D=0.1 \mathrm{~J}$ for $5 \times 10^{-11} \leq v / J \leq 10^{-7}, D=0.2 J$ and $0.3 J$ for $5 \times 10^{-10} \leq v / J \leq 10^{-7}$ and $0.01 \leq k_{B} T / J \leq 0.1$.

Table 3

Parameters of the fits and estimates of the switching field at $0 K$ and the energy barrier in zero field for a 33-spin particle with different values of the anisotropy constant.

\begin{tabular}{|l|l|l|l|}
\hline$D / J$ & 0.1 & 0.2 & 0.3 \\
\hline$H_{s w}^{0} / J$ & 0.215 & 0.430 & 0.645 \\
$c / k_{B}$ & $10^{-3}$ & $10^{-3}$ & $10^{-2}$ \\
axis intercept & 0.219 & 0.432 & 0.646 \\
slope & -0.132 & -0.207 & -0.252 \\
$\Delta E_{0} / J$ & 2.8 & 4.3 & 6.6 \\
$\tau_{0}$ & 40 & 50 & 5 \\
\hline
\end{tabular}

two fits are reported in TABLE 3 in comparison with the case $D=0.1 \mathrm{~J}$. As for $D=0.1 \mathrm{~J}$, a self-consistent fit is obtained with $H_{s w}^{0}$ slightly higher than the expected value but our estimates of $H_{s w}^{0}$ are proportional to the anisotropy constant in agreement with the Stoner-Wohlfarth model. The extracted $\Delta E_{0}$ is lower than the expected value again, the increase of the misfit with the anisotropy constant being attributed to the evolution towards a non-uniform rotation.

For $D=J$, it is not possible to put the low temperature data points on a single straight line. The best fit is shown in Fig. 9. Note that the switching field values are significantly lower than that predicted in the case of uniform rotation $\left(H_{s w}^{0} / J=2\right)$. Actually, at very low temperature, only spin flips 


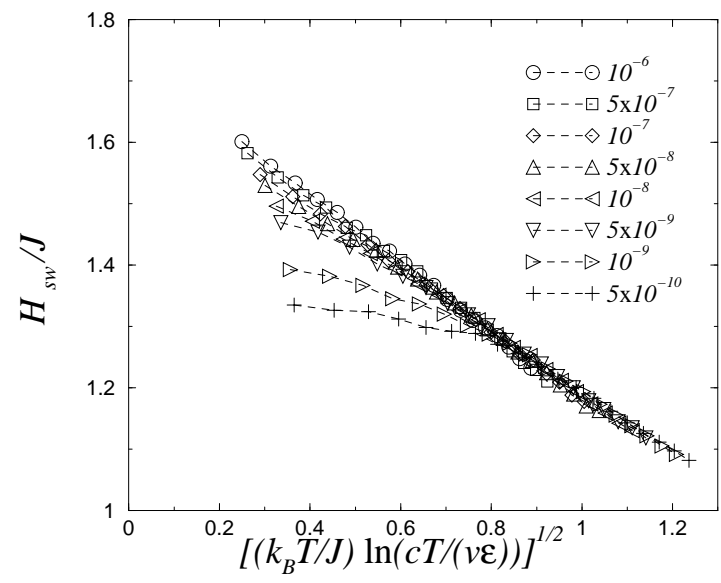

Fig. 9. Scaling plot of the mean switching field values of a 33 -spin particle with $D=J$ for $5 \times 10^{-10} \leq v / J \leq 10^{-6}$ and $0.01 \leq k_{B} T / J \leq 0.1$.

can occur, so the switching field does not depend anymore on the anisotropy constant but rather on the exchange interaction and the coordination number. We expect that the numerical estimate of the switching field at very low temperature would have not been changed increasing the value of the anisotropy constant.

\section{Conclusion}

Using a MC single spin rotation algorithm, we have realized the Néel-Brown model of the magnetisation reversal for nanoparticles with realistic values of $R / \lambda$ and $R / \delta$. By choosing the temperature and the field sweeping rate appropriately, it has been possible to check the validity of the approximated expression of the mean switching field. For reasonable anisotropy $(D \leq 0.3 J)$, the scaling behaviour is satisfied at very small field sweeping rates $\left(v / J \leq 10^{-8}\right)$ for all temperatures studied except the case $N=7$ and $D=0.1 \mathrm{~J}$ for which the superparamagnetic regime can be observed. On the other hand, for high anisotropic nanoparticles $(D=J)$, the magnetisation reversal is not uniform and the mean switching field values can not be fitted anymore following the proposed approximated expression.

Let us briefly remind that our estimates of $H_{s w}^{0}$ are independent of the size and vary linearly with the anisotropy constant, as expected. The discrepancy between the estimate of $\Delta E_{0}$ and its expected value in all cases might be due to the spin rotation procedure.

In a near future, it is planned to investigate the effect of physical parameters, such as surface anisotropy and dipolar interactions, in relation with the shape of the particle. However, one has to be aware of the very large increase of CPU time by taking into account the dipolar interactions.

\section{Acknowledgments}

This work was supported by the computer-time grants 2000006 of the Centre de Ressources Informatiques de Haute Normandie (CRIHAN) and C20020922345 of the Centre Informatique National de l'Enseignement Supérieur (CINES). We are indebted to W. Wernsdorfer and N. Lecoq for interesting discussions.

\section{References}

[1] C. Kittel, Rev. Modern Phys. 21, 541 (1949).

[2] C.B. Bean and J.D. Livingston, J. Appl. Phys. 30, 1205 (1959).

[3] A. Tasaki, S. Tomiyana and S. Iida, J. Appl. Phys. 4, 707 (1965).

[4] C. Hayashi, J. Vac. Sci. Technol. A5, 1375 (1987).

[5] A. Aharoni, Introduction to the theory of ferromagnetism (Oxford University Press, London, 2000).

[6] A.H. Morrish, The Physical Principles of Magnetism (John Wiley, New York 1965).

[7] W. Wernsdorfer et al, Phys. Rev. Lett. 78, 1791 (1997).

[8] W. Wernsdorfer et al, Phys. Rev. Lett. 79, 4014 (1997).

[9] M. Jamet et al, Phys. Rev. Lett. 86, 4676 (2001).

[10] W. Wernsdorfer, Adv. Chem. Phys. 118, 99 (2001).

[11] L. Néel, Ann. Geophys. 5, 99 (1949).

[12] W.F. Brown, Phys. Rev. 130, 1677 (1963).

[13] D. Garcia-Pablos, P. Garcia-Mochales, N. Garcia and P.A. Serena, J. Appl. Phys. 79, 6019 (1996).

[14] H.L. Richards, M.A. Novotny and P.A. Rikvold, Phys. Rev. B 54, 4113 (1996). 
[15] H.L. Richards et al, Phys. Rev. B 55, 11521 (1997).

[16] D. Hinzke and U. Nowak, Phys. Rev. B 58, 265 (1998).

[17] D. Hinzke and U. Nowak, Comp. Phys. Com. 121122, 334 (1999).

[18] U. Nowak, R.W. Chantrell, E.C. Kennedy, Phys. Rev. Lett. 84, 163 (2000).

[19] J. Kurkijärvi, Phys. Rev. B 6, 832 (1972).

[20] A. Garg, Phys. Rev. B 51, 15592 (1995)

[21] E.C. Stoner and E.P. Wohlfarth, Philos. Trans. Soc. London Ser. A 240, 599 (1948).

[22] R.H. Victora, Phys. Rev. Lett. 63, 457 (1989).

[23] I. Klik and L. Gunther, J. Stat. Phys. 60, 473 (1990).

[24] P. Hänggi, P. Talkner and M. Borkovec, Rev. Mod. Phys. 62, 251 (1990).

[25] A. Aharoni, J. Appl. Phys. 63, 5879 (1988).

[26] D.W. Heermann, Computer Simulation Methods in Theoretical Physics (2nd Edition, Springer-Verlag, 1990).

[27] K. Binder and D.W. Heermann, Monte Carlo Simulation in Statistical Physics (2nd Edition, Springer-Verlag, 1990).

[28] P.K. Mac Keown, Stochastic Simulation in Physics (Springer, 1997).

[29] N. Metropolis, A.E. Rosenbluth, M.N. Rosenbluth, A.H. Teller and E. Teller, J. Chem. Phys. 21, 1087 (1953). 